\title{
Where long noncoding RNAs meet DNA methylation
}

\author{
Cell Research (2014) 24:263-264. doi:10.1038/cr.2014.13; published online 31 January 2014
}

DNA methylation is a fundamental epigenetic mechanism governing regulation of gene expression during mammalian development. A recent study published in Nature shows a novel long noncoding RNA (IncRNA) arising from the $C E B P A$ gene locus (termed $e c C E B P A$ ) that is critical for regulation of DNA methylation at this site through the association of ec$C E B P A$ with DNA methyltransferase 1, DNMT1.

DNA methylation was the first discovered epigenetic mechanism [1, 2]. It is a chemical modification of genomic DNA by the addition of a methyl group (-CH3) to the cytosine or adenine DNA nucleotides. Typical DNA methylation occurs in a $\mathrm{CpG}$ dinucleotide context, where predominantly $\mathrm{CpG}$ sites are methylated in the genome. Most of the $\mathrm{CpG}$ clusters, known as $\mathrm{CpG}$ islands, occur near transcriptional start sites (TSSs) where they are predominantly un-methylated [3]. The establishment and maintenance of methylation patterns resulting in modulation of gene expression is one of the key steps in epigenetic regulation during normal developmental programs. In mammalian cells, DNA (Cytosine-5-)-Methyltransferase 1 (DNMT1) is the most abundant DNA methyltransferase to transfer a methyl group to DNA. It is highly expressed during the $\mathrm{S}$ phase, localizing to replication foci and interacting with the proliferating cell nuclear antigen (PCNA) to maintain DNA methylation marks during replication. This protein is known to add a methyl group to the un-methylated cytosines in methylCpG:CpG DNA from methylated parent and un-methylated daughter strands to generate fully methylated methyl-CpG: methyl-CpG pairs [4].

Long noncoding RNAs (lncRNAs) have been defined as transcripts of $>$ 200 nucleotides without protein coding capacity. Depending on their locus of expression, they can be categorized as intragenic or intergenic [5]. While recent genome-wide studies indicated that over $74 \%$ of human genome was transcribed, only $2 \%$ of these transcripts correspond to protein-coding genes [6]. While IncRNAs have been shown to have a broad range of biological functions both in the cytoplasm and the nucleus, a large number of nuclear lncRNAs were shown to originate from distal regulator elements (enhancers) to positively regulate gene expression [79]. Interestingly, while IncRNAs display tissue-specific expression patterns [10], they have a low evolutionary conservation rate between species [11].

In a recent paper published in Nature, Di Ruscio and colleagues reported the association of DNMT1 with a lncRNA termed ecCEBP (extra-coding CEBPA) expressed from the $C E B P A$ locus. This transcript was shown to initiate about $2 \mathrm{~kb}$ from the CEBPA TSS in the sense direction and extend nearly $3 \mathrm{~kb}$ beyond the $C E B P A$ mRNA. While CEBPA is a polyadenylated transcript with a predominant cytoplasmic localization, ecCEBP is not polyadenylated and is enriched in the nuclear fraction. Interestingly, while the authors show a concordant expression of both transcripts in all tested human tissues, the ecCEBP synthesis occurs prior to expression of CEBPA in $\mathrm{S}$ phase of the cell cycle. To gain insight into the biological function of ecCEBPA, the authors depleted ec-
CEBPA transcripts using multiple short hairpin RNAs (shRNAs) against the 3'end of the noncoding transcript beyond the $3^{\prime}$-UTR of the CEBPA gene in a U937 cell line. Depletion of ecCEBPA resulted in a concomitant decrease in the CEBPA mRNA, suggestive of an activating role for this noncoding RNA. Since $C E B P A$ gene is subject to silencing by DNA methylation [12], the authors assessed whether depletion of ecCEBPA resulted in changes in DNA methylation of $C E B P A$ distal promoter. Interestingly, they observed a robust increase in levels of DNA methylation of CEBPA promoter region following the depletion of noncoding transcript. Additionally, overexpression of a downstream region of ecCEBPA (termed R1 region) had the opposite effect in K562 cells, resulting in increased expression of $C E B P A$ and substantial reduction of DNA methylation across the CEBPA locus. Indeed, genome-wide analysis of the effects of R1 overexpression revealed that a small number of genomic loci (3.3\%) displayed changes in DNA methylation comparable to that of $C E B$ $P A$ locus, supporting a specific function for ecCEBPA in DNA methylation.

Since ecCEBPA transcripts peaks at $\mathrm{S}$ phase, coincident with increased DNMT1 expression and enzymatic activity, the authors assessed whether ecCEBPA and DNMT1 physically associate. RNA immuoprecipitation (RIP) analysis suggested the interaction of nonpolyadenylated ecCEBPA transcript and DNMT1. Next the authors performed a detailed analysis of DNMT1 association with different domains of ecCEBP transcript and its mutant constructs using RNA electrophoresis 
mobility shift assays (REMSA). These experiments revealed the interaction of DNMT1 with RNA domains that assumed a stem-loop structure. Moreover, they found a stronger affinity between DNMT1 and stem-loop structured RNAs than un-methylated or methylated DNA. Moreover, they were able to map the RNA binding interface to a region encompassing the DNMT1 catalytic domain. These experiments revealed the preference of DNMT1 association with structured RNA motifs.

To assess the role of RNA in regulation of DNMT1 enzymatic activity, the authors devised an elegant assay fusing a hemi-methylated DNA (bottom strand methylated) to downstream of the T7 RNA polymerase promoter where DNA methylation of the upper strand could be measured. They show that while in the absence of T7 RNA polymerase there was ample DNA methylation, addition of RNA polymerase, resulted in induction of transcription and inhibition of DNA methylation. Moreover, addition of ribo-oligonucleotides to a DNA methylation reaction using DNMT1 inhibited the catalytic activity of the enzyme. These results collectively demonstrated a potent regulation of DNMT1 enzymatic activity by RNA. Finally, the authors performed RIP fol- lowed by high throughput sequencing to assess the genome-wide association of DNMT1 with RNA. Interestingly, the majority of DNMT1-interacting RNAs were deemed to be non-polyadenylated. Moreover, they found a negative correlation between DNMT1-RNA association and the methylation status of a particular locus, supportive of the global function of RNA in DNMT1 regulation.

Taken together, the authors propose a model in which a class of nonpolyadenylated lncRNAs by virtue of their association with DNMT1 regulate DNA methylation patterns. This is an intriguing proposal for regulation of an important epigenetic modification in mammalian cells. Regulation of DNA methylation by lncRNAs could be an important mechanism for regulation of gene expression during development and disease. This mechanism is particularly relevant at distal regulatory elements (enhancers) where expression of non-polyadenylated enhancer RNAs could regulate the DNA methylation status of enhancer sites. While this study was focused on DNMT1, it is likely that other DNA methyltransferase enzymes (DNMT3a, DNMT3b) may also display an association with lncRNAs, which may regulate their enzymatic activity and modulate the patterns of de novo
DNA methylation. Indeed, these are exciting times for studies of epigenetic mechanisms and the new regulatory roles for noncoding RNAs in epigenetic regulation.

\section{Fan Lai ${ }^{1}$, Ramin Shiekhattar ${ }^{1}$ \\ ${ }^{1}$ Wistar Institute, Philadelphia, PA 19104, USA \\ Correspondence: Ramin Shiekhattar \\ E-mail: Shiekhattar@wistar.org}

\section{References}

1 Holliday R, JE Pugh. Science 1975, 187: 226-232.

2 Riggs AD. Cytogenet Cell Genet 1975; 14:9-25.

3 Bird AP. Nature 1986; 321:209-213.

4 Hermann A, Goyal R, Jeltsch A. J Biol Chem 2004; 279:48350-48359.

5 Mercer TR, Dinger ME, Mattick JS. Nat Rev Genet 2009; 10:155-159.

6 Djebali S, Davis CA, Merkel A, et al. $\mathrm{Na}$ ture 2012; 489:101-108.

7 Lai F, Orom UA, Cesaroni M, et al. Nature 2013; 494:497-501.

8 De Santa F, Barozzi I, Mietton F, et al. PLoS Biol 2010; 8:e1000384.

9 Wang KC, Yang YW, Liu B, et al. Nature 2011; 472:120-124.

10 Mercer TR, Gerhardt DJ, Dinger ME, et al. Nat Biotechnol 2012; 30:99-104.

11 Wang J, Zhang J, Zheng $\mathrm{H}$, et al. Nature $2004 ; \mathbf{4 3 1}$ : 1 p following 757 ; discussion following 757.

12 Hackanson B, Bennett KL, Brena RM, et al. Cancer Res 2008; 68:3142-3151. 